\title{
Neonatal Thrush Is Not Associated with Mode of Delivery
}

\author{
Tanen T. St. Clair-Brown, DO, Kaitlin E. Schwerer, DO, and Godwin Y. Dogbey, PhD
}

Purpose: Thrush is an opportunistic infection of the buccal cavity by the organism Candida albicans. It is most commonly seen in infants and becomes relatively uncommon between 6 to 9 months of age implying the infection is possibly dictated by risk factors present at or around the time of birth. The objective of this study is to determine if there is an association between the development of thrush and cesarean delivery, as we hypothesize.

Methods: Neonatal records were obtained through retrospective chart review of the past 10 years from an outpatient clinic and hospital records in Sandusky Ohio. ICD 9 and 10 codes for thrush and well child visit were used to identify patients. Statistical analysis performed on the data included: multivariable logistic regression, $\chi^{2}$ test of association, and nonparametric $\chi^{2}$ test of goodness-of-fit.

Results: A total of 636 patient records were reviewed, with 127 having a diagnosis of thrush and 509 without the diagnosis. A multivariable logistic regression indicated that during the first year of life, children born by cesarean section were no more likely to develop thrush compared with those born by vaginal delivery, controlling for age and sex of the neonate as well as parity and group B streptococcus status of the mother.

Conclusion: This study demonstrated that there is no significant correlation between the mode of delivery and the development of thrush. It is reasonable to conclude that of the many factors associated with neonatal thrush development, mode of delivery is likely not one of them. ( $\mathrm{J}$ Am Board Fam Med 2018;31:537-541.)

Keywords: Caesarean Section, Infant, Logistic Regression, Ohio, Obstetric Delivery, Opportunistic Infections, Oral Candidiasis, Pregnancy

Thrush is an opportunistic infection of the buccal cavity by the commensal organism Candida albicans. Colonization by Candida yeast is thought to occur at an early age, with the organism being acquired during passage through the birth canal, nursing, or from food. There are approximately 200 species in the genus Candida and within this group, C. albicans accounts for nearly $75 \%$ of all candidal infections. Thrush occurs, in part, when innate and acquired host-defense mechanisms do not act in concert

This article was externally peer reviewed.

Submitted 31 October 2017; revised 24 January 2018; accepted 25 January 2018.

From Firelands Regional Medical Center, Sandusky, OH (TTSC-B, KES); Ohio University Heritage College of Osteopathic Medicine, Athens (GYD).

Funding: none.

Conflict of interest: none declared.

Corresponding author: Kaitlin E. Schwerer DO, Firelands Regional Medical Center, 1111 Hayes Avenue, Sandusky, Ohio 44870 (Email: kaitlinschwerer@gmail.com). with the resident bacterial flora. This leads to an imbalance within the system, providing an ecological advantage for $C$. albicans to flourish. ${ }^{1}$ In most cases, thrush is limited to the neonatal and infant periods of life. Thrush is also associated with immunocompromised or polyendocrine disorders as well as iatrogenic causes, such as antibiotic or steroid use. Thrush is seen in as many as $37 \%$ of newborns during the first month of life and is associated, internationally, with poorly nourished populations. $^{2}$ In mild cases, the disease is typically self-limiting but is associated with discomfort; this becomes concerning in newborns, as it can affect their ability to feed. Thrush occurs equally in males and females. ${ }^{2}$ The disease is rare during the first week of life, with incidence peaking around the fourth week of life; it becomes uncommon after 6 months to 9 months of age once the child's immune system has established itself. ${ }^{2}$ Thrush, however, can occur at any age in predisposed patients. 
Due to the temporal nature of thrush, along with its predilection of the neonate without gender specificity, it is thought that this disease may, in part, be influenced by disruption in the infant's oral microbiota. ${ }^{1}$ There has been research demonstrating that there is a difference between the oral bacteria of vaginally-delivered versus cesarean-delivered neonates. ${ }^{3}$ There is considerable research on the microbiome of the neonate's intestines, along with several studies that have correlated a difference between the intestinal microbiota of vaginally-delivered versus cesarean-delivered neonates. ${ }^{4,5,6,7}$ Mode of delivery has also shown association with differences in the occurrence of atopic diseases, such as eczema and asthma $a^{8,9,10}$ as well as inflammatory bowel disease ${ }^{11}$ and obesity. ${ }^{12}$ Therefore, these known findings suggest the oral microbiota, which is influenced by the mode of delivery, could possibly influence the neonate's susceptibility to oral candidal stomatitis. To our knowledge there is only 1 study that looked at the association between the mother's vaginal colonization with candida at the time of birth and the neonates development of thrush. This study explored the direct transfer of candida to the neonate and did not look into whether the development of thrush, or lack thereof, was associated with the transfer of protective flora from the mother. ${ }^{13}$ The goal of this study was to investigate the association of mode of delivery and the development of thrush within the first 12 months of life.

\section{Methods}

Institutional review board exemption was obtained based on the retrospective nature of this study and the removal of identifiable information by the electronic medical records during the initial data collection, leaving behind medical record number, age, sex, mother's parity, group B streptococcus

Table 1. International Classification of Diseases Codes Used in Study

\begin{tabular}{lll}
\hline & Thrush & Well Visit \\
\hline ICD-10 & \multirow{2}{*}{112.0} & Z00.110 \\
& & Z000.111 \\
& & Z000.121 \\
ICD-9 & B37.0 & V20.2 \\
& & V20.31 \\
& & V20.32 \\
\hline
\end{tabular}

(GBS) status, and thrush ICD codes. Data were collected from Family Health Services of Erie County (FHS) and birth records from Firelands Regional Medical Center, both located in Sandusky, Ohio.

Records from the past 10 years at FHS and ICD codes (Table 1 ) were used to identify study participants, due to lack of an electronic medical record before 2007. ICD 9 and 10 codes for oral candidiasis were used to identify patients who had a diagnosis of thrush before 12 months of age and each child was only counted once. The mode of delivery in these patients was obtained from birth records from Firelands Regional Medical Center. Other variables, age and sex of the neonates as well as the mother's parity and GBS status, were also obtained and explored as potential confounding variables.

The control group was selected from the FHS population by using ICD 9 and 10 codes (Table 1) for well-child visits; this generated 2391 total potential patients. The data were again refined to 509 randomly selected patients, with the use of $\mathrm{Mi}$ crosoft Excel's randomization function. This was done to achieve a strong statistical power by using a 1:4 ratio of thrush to control while compromising for time consideration of the study.

Twelve months of age was used as the cut off in selection criteria, because maternal protection bestowed to the neonate starts to wane around the 6-month mark and is reasonably gone by 12 months of age. ${ }^{14}$ Furthermore, long-term colonization is probably responsible for eliciting the circulating immunoglobulin-G (IgG) and mucosal secretory immunoglobulin-A (S-IgA) antibodies to $C$. albicans that are detectable in most healthy individuals. It is likely these host defenses, along with circulating leukocytes and macrophages, help restrict $C$. albicans superficial growth on the mucosal surfaces. ${ }^{1}$ Lastly, 12 months is the point in time when infants, in the United States, start to receive their first live-attenuated vaccine. ${ }^{15}$ For the purposes of this study, we consider the adaptive immune system to be working in individuals by this point in time due to the delivery of live vaccines.

Gestational age of less than 34 weeks was excluded in both groups due to prematurity being a risk factor for a lower birth weight, which correlates with an increased risk of oral candidal infections. All neonates in this study were well above the cut off of $2000 \mathrm{~g}$ at birth, which is what most articles reference as a risk factor for oral candidia- 
Table 2. Thrush Population Characteristics

\begin{tabular}{lcc}
\hline & Male $(\mathrm{n}=68)$ & Female $(\mathrm{n}=59)$ \\
\hline Cesarean section birth & 25 & 13 \\
Vaginal birth & 43 & 46 \\
GBS+ & 13 & 12 \\
GBS- & 36 & 28 \\
Unknown GBS status & 18 & 19 \\
Nulliparous & 15 & 22 \\
Multiparous & 41 & 22 \\
Unknown parity & 12 & 15 \\
\hline
\end{tabular}

GBS, group B streptococcus.

sis. ${ }^{16,17,18}$ Mode of delivery, vaginal versus cesarean section, was used as the exposure arm of the study.

Various statistical methods were used in this study. These included the multivariable logistic regression, unadjusted $\chi^{2}$ test of association, and nonparametric $\chi^{2}$ test of goodness-of-fit. Statistical significance was evaluated at $P \leq .05$. Variables also of interest included age, sex, mother's parity, and GBS status, which were also used for subanalysis regarding thrush risk. It was assumed that a positive GBS status in the mother indicated antepartum antibiotic use. Due to the retrospective design of this study, some data could not be obtained. If the mode of delivery could not be determined, these neonates were excluded from the study. Data of the mother's parity and GBS status could not be completely accounted for, as seen in Table 2 and Table 3.

\section{Results}

In all, 636 patients' medical records were obtained and reviewed (Table 2, Table 3). Of these, 127 participants who were all greater than 34 weeks of gestation at birth were identified with a diagnosis of

Table 3. Well-Child Population Characteristics

\begin{tabular}{lcc}
\hline & Male $(\mathrm{n}=260)$ & Female $(\mathrm{n}=249)$ \\
\hline Cesarean section birth & 97 & 67 \\
Vaginal birth & 163 & 182 \\
GBS + & 42 & 44 \\
GBS- & 122 & 112 \\
Unknown GBS status & 96 & 93 \\
Nulliparous & 64 & 56 \\
Multiparous & 122 & 124 \\
Unknown parity & 74 & 69 \\
\hline
\end{tabular}

GBS, group B streptococcus. thrush before 12 months of age. The unadjusted $\chi^{2}$ analysis showed no statistically significant association between thrush and mode of delivery $(P=$ .619). (Table 4) This result was also consistent with that obtained from a multivariable logistic regression that indicated that during the first year of life, children born by cesarean section were no more likely to develop thrush compared with those born by vaginal delivery $(P=.533$; odds ratio $[\mathrm{OR}]$, 1.190; 95\% CI, 0.688-2.06) (Table 5), controlling for the effects of age and sex of the neonate and the parity and GBS status of the mother. One intriguing result, however, was that neonate sex was marginally predictive of mode of delivery $(P=.032$; OR, 1.64; 95\% CI, 1.04-2.57) (Table 5). That is, male neonates were more likely to be delivered vaginally when compared with female neonates.

\section{Discussion}

Our study found that there is no significant association between the mode of delivery and the development of thrush in the first year of life. Through extensive literature searches, we have been unable to find any previous studies that support or refute these findings. Current knowledge of thrush does demonstrate many models as to why $C$. albicans can become an opportunistic infection of the buccal cavity. It is reasonable to conclude that the development of thrush is multifactorial; however, mode of delivery likely does not contribute, based on these findings.

Although it should be noted that in other studies thrush is known to peak around the fourth week of life, ${ }^{2}$ this study may reflect only the prethreshold relationship between mode of delivery and the incidence of thrush (eg, before 12 months of age). Carving out the threshold or postthreshold relationships would require extending the study to a later age of life.

Furthermore, these results showed a marginal association between child's sex and mode of delivery. Other reports have noted a higher relative risk of cesarean sections with male neonates; this research would contradict this notion. ${ }^{19}$ We would consider this a spurious correlation in the sense of it being statistically significant but perhaps clinically insignificant in its practical application. This said, there may be the need to explore this phenomenon further to conclusively establish if indeed this observed association was purely due to chance or a real development worthy of clinical attention. 
Table 4. Statistical Tests

\begin{tabular}{lccccc}
\hline & Value & $d f$ & $\begin{array}{c}\text { Asymptotic Significance } \\
(2 \text {-sided })\end{array}$ & $\begin{array}{c}\text { Exact Significance } \\
\text { (2-sided) }\end{array}$ & $\begin{array}{c}\text { Exact Significance } \\
(1 \text {-sided })\end{array}$ \\
\hline Pearson $\chi^{2}$ & 0.248 & 1 & .619 & \\
Continuity correction & 0.153 & 1 & .696 & .617 & .350 \\
Likelihood ratio & 0.250 & 1 & .619 & \\
Fisher's exact test & & & .619 & \\
Linear-by-linear association & 0.247 & 1 & & \\
Valid cases, N & 636 & & & \\
\hline
\end{tabular}

Table 5. Variables in the Equation

\begin{tabular}{|c|c|c|c|c|c|c|c|c|}
\hline & \multirow[b]{2}{*}{ B } & \multirow[b]{2}{*}{$\mathrm{SE}$} & \multirow[b]{2}{*}{ Wald } & \multirow[b]{2}{*}{$d f$} & \multirow[b]{2}{*}{ Sig.* } & \multirow[b]{2}{*}{ Exp\# } & \multicolumn{2}{|c|}{$95 \%$ CI for Exp } \\
\hline & & & & & & & Lower & Upper \\
\hline Thrush & 0.174 & 0.280 & 0.388 & 1 & 0.533 & 1.190 & 0.688 & 2.060 \\
\hline Sex & -0.494 & 0.230 & 4.620 & 1 & 0.032 & 0.610 & 0.389 & 0.957 \\
\hline Parity & -0.321 & 0.246 & 1.708 & 1 & 0.191 & 0.725 & 0.448 & 1.174 \\
\hline GBS & -0.034 & 0.258 & 0.017 & 1 & 0.895 & 0.967 & 0.583 & 1.602 \\
\hline Constant & -0.757 & 0.337 & 5.054 & 1 & 0.025 & 0.469 & & \\
\hline
\end{tabular}

CI, confidential interval; GBS, group B streptococcus; SE, standard error.

*Sigma.

\#Exponential distribution.

The strengths of this study include ${ }^{1}$ a population-based retrospective study ${ }^{2}$, a relatively selfcontained unified health care system ${ }^{3}$, ascertainment of subjects by applying predetermined criteria through a comprehensive medical record review, and $^{4}$ reasonable statistical power because of the moderately large sample size.

Nonetheless, there are also inherent limitations of this study because of its retrospective design. The incidence of thrush may have been underreported in the medical records, potentially with less severe cases going unmentioned. Potential confounding variables were not included in the model, such as exposure to day care, siblings, or family members who may have transmitted the candidal species to the patient. Oral candidiasis has been reported to be strongly associated with socioeconomic status, which was not taken into consideration in this study. The studied population likely mimicked the population of Erie County, Ohio, where FHS and Firelands Regional Medical Center are located. The local population is predominantly white/not Latino (83.7\%) and is not representative of the US population as a whole. ${ }^{20}$ It was also assumed that all children in the study have an adequate innate immune system, which may have not been the case. The main limitation of this study was in the data collection process; the diagnosis of thrush was only captured if the patient presented to FHS, an outpatient clinic, not to the emergency department. It was also difficult to find a structured way to obtain a control population; therefore, wellchild visits were used, which captured most of the children within FHS, spanning over the past 10 years.

This study looked at infants with a diagnosis of thrush within the first 12 months of life and the relationship to vaginal or cesarean delivery. Other studies have shown differences in oral and gut microbiota as well as varying potential for asthma, eczema, and obesity based on the mode of delivery. As a result of this study, we conclude that it does not seem that mode of delivery has an impact on the development of thrush within the first 12 months of life, but further research is warranted.

To see this article online, please go to: http://jabfm.org/content/ 31/4/537.full.

\section{References}

1. Jenkinson HF, Douglas LJ. Interactions between Candida species and bacteria in mixed infections. In: Brogen KA, Guthmiller, JM, editors. Polymicrobial Diseases. Washington DC: ASM Press; 2002. PP. 357-74. 
2. Kumar, M. Thrush. Background, pathophysiology, epidemiology. Available from: http://emedicine. medscape.com/article/969147-overview. Published January 6, 2017. Accessed September 26, 2016.

3. Barfod MN, Magnusson K, Lexner MO, Blomqvist S, Dahlén G, Twetman S. Oral microflora in infants delivered vaginally and by caesarean section. Int $\mathrm{J}$ Paediatr Dent 2011;21:401-6.

4. Grölund M-M, Lehtonen O-P, Eerola E, Kero P. Fecal microflora in healthy infants born by different methods of delivery: permanent changes in intestinal flora after cesarean delivery. J Pediatr Gastroenterol Nutr 1999;28:19-25.

5. Brumbaugh DE, Arruda J, Robbins K, et al. Mode of delivery determines neonatal pharyngeal bacterial composition and early intestinal colonization. J Pediatr Gastroenterol Nutr 2016;63:320-8.

6. Rautava S, Luoto R, Salminen S, Isolauri E. Microbial contact during pregnancy, intestinal colonization and human disease. Nat Rev Gastroenterol Hepatol 2012;9:565-76.

7. Biasucci G, Benenati B, Morelli L, Bessi E, Boehm G. Cesarean delivery may affect the early biodiversity of intestinal bacteria. J Nutr 2008;138:1796$1800 \mathrm{~S}$.

8. Mueller NT, Bakacs E, Combellick J, Grigoryan Z, Dominguez-Bello MG. The infant microbiome development: mom matters. Trends Mol Med 2015;21: $109-117$.

9. Nimwegen FAV, Penders J, Stobberingh EE, et al. Mode and place of delivery, gastrointestinal microbiota, and their influence on asthma and atopy. J Allergy Clin Immunol 2011;128:948-55.

10. Penders J, Thijs C, Brandt PAVD, et al. Gut microbiota composition and development of atopic manifestations in infancy: the KOALA Birth Cohort Study. Gut 2007;56:661-7.

11. Khalili H, Ananthakrishnan AN, Higuchi LM, Richter JM, Fuchs CS, Chan AT. Early life factors and risk of inflammatory bowel disease in adulthood. Inflamm Bowel Dis 2013;19:542-7.

12. Ajslev TA, Andersen CS, Gamborg M, Sørensen TIA, Jess T. Childhood overweight after establishment of the gut microbiota: the role of delivery mode, pre-pregnancy weight and early administration of antibiotics. Int J Obes 2011;35:522-9.

13. Al-Rusan RM, Darwazeh AM, Lataifeh IM. The relationship of Candida colonization of the oral and vaginal mucosae of mothers and oral mucosae of their newborns at birth. Oral Surg, Oral Med, Oral Pathol, Oral Rad 2017;123:459-63.

14. Niewiesk S. Maternal antibodies: clinical significance, mechanism of interference with immune responses, and possible vaccination strategies. Front Immunol 2014;5.

15. Centers for Disease Control and Prevention. Measles, mumps, and rubella (MMR) vaccine safety. Available from: https://www.cdc.gov/vaccinesafety/ vaccines/mmr-vaccine.html. Published May 4, 2017. Accessed January 8, 2017.

16. Tinoco-Araujo JE, Araújo DF, Barbosa PG, Santos PS, Medeiros AM. Invasive candidiasis and oral manifestations in premature newborns. Einstein (Sao Paulo, Brazil) 2013;11:71-5.

17. UCSF Children's Hospital. Intensive Care Nursery House Staff manual: Candidiasis in the Newborn. Available from: https://www.ucsfbenioff childrens. org/pdf/manuals/44[lowern]Candidiasis.pdf. Published 2004. Accessed January 5, 2017.

18. Kaufman, DA. Fungal infections in preterm infants. Available from: http://emedicine.medscape.com/ article/980487-overview. Published March 22, 2017. Accessed January 8, 2017.

19. Renzo GCD, Rosati A, Sarti RD, Cruciani L, Cutuli AM. Does fetal sex affect pregnancy outcome? Gender Med 2007;4:19-30.

20. U.S. Census Bureau. QuickFacts: Erie County, Ohio. Available from: https://www.census.gov/ quickfacts/table/PST045215/39043. Accessed May 6, 2017. 